\section{Questión}

Periodismo / Comunicación

ISSN 1669-6581

La mujer como periodista especializada en deporte. Caso provincia de Loja, Ecuador

Sybel Enrique Ontaneda Andrade, César Teodomiro Sandoya Valdiviezo, Hever Sánchez

Question/Cuestión, Vol. 2, N 66, Agosto 2020

ISSNe 1669-6581

https://perio.unlp.edu.ar/ojs/index.php/question/index

IICom-FPyCS-UNLP

DOI: https//doi.org/10.24215/16696581e508

\title{
La mujer como periodista especializada en deporte. Caso provincia de Loja, Ecuador
}

Women as journalist specialized in sports. Case province of Loja, Ecuador

Sybel Enrique Ontaneda Andrade

Facultad de Educación, Arte y Comunicación, Universidad Nacional de Loja,

Ecuador

sybel.ontaneda@unl.edu.ec

https://orcid.org/0000-0001-5947-6394

César Teodomiro Sandoya Valdiviezo

Facultad de Educación, Arte y Comunicación, Universidad Nacional de Loja,

Ecuador 
cesar.sandoya@unl.edu.ec https://orcid.org/0000-0002-9343-155X

Hever Sánchez Martínez

Facultad de Educación, Arte y Comunicación, Universidad Nacional de Loja, Ecuador

hever.sanchez@unl.edu.ec https://orcid.org/0000-0001-5189-5164

\section{Resumen}

El presente estudio busca conocer y analizar las causas que condicionan que las mujeres periodistas de la ciudad ecuatoriana de Loja no se especialicen en el periodismo deportivo, pese a la existencia de medios de comunicación que abordan el tema en secciones impresas o programas de radio y televisión. Se asume una perspectiva cualitativa mediante el empleo de métodos investigativos como el científico, el inductivo y el deductivo y la aplicación de técnicas como la encuesta a ciudadanos lojanos y la entrevista a directivos de programas especializados en deporte. La investigación concluye que la existencia de estereotipos sobre el rol de la mujer en el periodismo que aún no han sido superados, la escasa preparación recibida en la academia para familiarizarse con referentes teórico-prácticos sobre el tema, la falta de apoyo por parte de los directores y productores de programas deportivos y la insuficiente remuneración económica con respecto a la que reciben los hombres, figuran entre las principales causas que desmotivan a las mujeres periodistas a especializase en la información deportiva. 


\title{
Palabras clave
}

Periodismo especializado, periodismo deportivo, equidad de género, programa informativo.

\begin{abstract}
The present study seeks to know and analyze the causes that condition that women journalists in the Ecuadorian city of Loja do not specialize in sports journalism, despite the existence of media that address the issue in printed sections or radio and television programs. A qualitative perspective is assumed through the use of research methods such as scientific, inductive and deductive and the application of techniques such as the survey of distant citizens and the interview with managers of specialized sports programs. The investigation concludes that the existence of stereotypes about the role of women in journalism that have not yet been overcome, the poor preparation received in the academy to familiarize themselves with theoretical-practical references on the subject, the lack of support from the directors and producers of sports programs and the insufficient economic remuneration with respect to which men receive, are among the main causes that discourage women journalists to specialize in sports information.
\end{abstract}

\section{Key words}

Specialized journalism; Sports journalism; gender equality; informational program 


\section{Introducción}

A lo largo del siglo XX la mujer logró empoderarse en distintos espacios dentro de la esfera social. El ámbito del periodismo fue uno de ellos, en los que cobró mayor fuerza la presencia femenina fundamentalmente después de la década de los 40 , cuando la radio se había posesionado como medio de comunicación y la televisión daba pasos incipientes.

Hubo esferas dentro de la actividad periodística en las que las mujeres lograron especializarse como la moda o la salud; sin embargo, otras como el área deportiva se pensó hasta hace un tiempo que era exclusiva de los hombres. No obstante, con el paso del tiempo "la mujer se fue ganando un espacio dentro de la comunicación deportiva, por sus conocimientos de los diversos eventos deportivos que suceden en el mundo" (Malla, 2017, p. 3). Y es que como afirma Moreno (2010), "el periodismo deportivo con la presencia de la mujer permitirá romper esquemas tradicionales, refrescando los sentidos de las masas, logrando así una justa y anhelada participación equitativa" (p. 26). Sin embargo, aunque existen en muchos medios de comunicación de todo el mundo mujeres periodistas especializadas en deporte, es notable un desequilibrio con respecto a los hombres.

En el caso concreto de la ciudad ecuatoriana de Loja, el periodismo deportivo comenzó en sus inicios a tener presencia en los medios impresos, que eran los más populares entonces para el target local (Kirby, 2008, p. 55). Actualmente, los medios audiovisuales presentan también propuestas deportivas que difunden hechos noticiosos de diferentes disciplinas deportivas; aunque muchos "sólo dan cabida al tema profesional dejando de lado el amateur" (Malla, 2017, p. 20). Estos formatos se caracterizan además por formar a la ciudadanía desde la apropiación de una educación física y una conciencia 
multideportiva que les permita, a largo plazo, tener conocimientos profundos sobre el tema (González, 2013).

Sin embargo, la presencia de la mujer en los medios de comunicación lojanos para reportar, comentar o presentar temas sobre deporte, es prácticamente nula. De ahí que este estudio tenga como objetivo general analizar las principales causas por las que las periodistas lojanas no se especializan en el periodismo deportivo.

Se formularon además como objetivos específicos: 1) sistematizar conceptos teóricos que asistan al análisis de la relación entre periodismo deportivo y equidad de género y 2) determinar los motivos por los que las periodistas no optan por el periodismo deportivo en los medios de comunicación de la localidad.

\section{Fundamentos teóricos. El periodismo especializado en deporte}

Desde la antigüedad hasta nuestros días, la dimensión que ha alcanzado el deporte lo ha convertido en "el fenómeno cultural con mayor desarrollo y centro de la esfera humana" (Lara, 2007, p. 77), gracias fundamentalmente a la acción de los medios de comunicación. Aunque por una parte estos realzan a los deportistas como reyes mediáticos, existe un sector que impone lógicas y criterios contrarios a esa presentación de los atletas como héroes (Moragas, 2008 , p. 16), que pondera los beneficios del deporte como actividad física, otorgando gran responsabilidad a las instituciones deportivas en el mantenimiento del equilibrio del sistema y la autonomía.

Por estas razones, Alcoba (2005) reconoce que el deporte es una actividad humana natural que por su trascendencia y aceptación social se ha hecho compleja, por lo cual está estrechamente relacionada con el conocimiento o los 
intereses que las audiencias y/o aficionados demuestren sobre determinada disciplina.

Desde una postura más sociológica, Billings (2010) reconoce que

El deporte puede ser un vehículo para entender a gente que nunca hemos conocido, así como también para vivir emociones que jamás hemos sentido. Con el incremento de los efectos de interacción parasocial dentro de los medios de comunicación masivos, los aficionados dan a conocer sus opiniones con un creciente sentido de empatía, compasión y testimonio que puede tener influencia en la manera en que procesan los mensajes recibidos, así como también en la manera en que formulan los mensajes emitidos hacia otros aficionados y entidades mediáticas. (p. 120)

Con la anterior cita, puede asegurarse que el periodismo deportivo sin duda contribuye a la interacción social, pues a través de este se puede organizar y mover a las masas debido al número de perceptores a los que llega y por la tecnología que se emplea en la transmisión y difusión de las noticias deportivas.

No cabe dudas entonces de que el deporte, como fenómeno cultural, es una parte muy importante del trabajo periodístico, por lo que debe tratarse como un género que da respuesta al interés que muestran las personas. La información relacionada con los eventos deportivos tiene mucha importancia para la comunidad, al extremo de que un medio de comunicación que no presente contenido deportivo, no tiene trascendencia para el colectivo. Para Paniagua Santamaría (2003), la información deportiva es, junto con la información económica, una de las áreas con mayor nivel de especialización a la que hay que darle una gran preparación. Esto se debe a que como fundamenta Alcoba (2010), los acontecimientos deportivos se vinculan estrechamente a otros 
sectores como la ciencia, la economía, la política o la sociedad misma. Esta diversidad que entraña el deporte, "convierte al periodismo especializado en un género súper especializado” (p. 203).

$Y$ es que en el caso del periodismo deportivo debe concebirse una exégesis de los hechos, estudiarse la interacción social que construye este gran prototipo, investigarse sus organizaciones o instituciones: la economía de los equipos, la sociología del hincha, el comportamiento de las masas televisivas, su globalización y su influencia en la sociedad. Para esto se demanda preparación y conocimiento especializado, pues este tipo de periodismo constituye el vínculo del progreso común entre medios y deporte. Es la red que los ata, que acerca a las audiencias a ambos y sostiene un espectáculo económico, social y cultural en evolución constante (Molina, 2009, p. 5).

Al reflejar mediáticamente cuanto se genera en materia deportiva, este periodismo se muestra como "un área peculiar, de gran seguimiento y con gran carga de emotividad" (Sobrados, 2005, p. 185), de ahí que cuente con un lenguaje específico que ha sido objeto de diversos análisis y estudios.

En sentido general, el lenguaje periodístico guarda estrecho vínculo con la lengua común y los intercambios entre estos se dan continuamente, porque los medios aglutinan un conjunto de subcódigos dentro de un código común donde conviven todas las variedades de la lengua oral y escrita (Hernández, 2003, p. 185). Muchos vocablos especializados pasan a ser comunes si el comunicador los emplea y extiende su uso y, en el caso concreto del deporte, si los hace comprender a los aficionados, pues el lenguaje periodístico especializado en deporte requiere cierto aprendizaje y dominio de la técnica, se caracteriza también por no participar del eufemismo, carecer de ambigüedades y ser 
directo, atrevido, claro, preciso y conciso, a fin de que las personas puedan comprenderlo de la mejor manera.

Según Moragas (2008) el lenguaje deportivo tiende a la espectacularización, "a una épica que, generalmente, define la propia identidad por la exclusión del contrario, sublimando la victoria y la derrota como expresiones del éxito, o del fracaso de la colectividad" (p. 23). Esto pudiera deberse a que, como documenta Hernández (2003, p. 14), el deporte es un fenómeno sociopolítico de gran alcance y a través del lenguaje deportivo se visualiza el espectáculo que influye enormemente en nuestra lengua hablada y escrita.

$\mathrm{Si}$ en un principio los medios contrataban como comentaristas o periodistas a deportistas que eran buenos comunicadores o a personas aficionadas y apasionadas por un fenómeno que se ha convertido en un género atractivo y que atrae al público, para seguir ganando seguidores del género femenino, en nuestros días hay mujeres que practican el periodismo deportivo.

\section{Equidad de género en el periodismo deportivo}

A lo largo de la historia, en todo el mundo y en la mayoría de culturas, las mujeres han sido sometidas a estructuras patriarcales regidas por leyes antiguas o religiones como el cristianismo y el islamismo, que constituyen antecedentes de los sistemas modernos que les han negado sus derechos humanos fundamentales, provocando la dependencia de la mujer, de forma análoga a la esclavitud, a la explotación de las clases desfavorecidas y a la mano de obra.

Un proyecto investigativo del Centro de Investigaciones de Estudios Sociales para América Latina (CIESPAL) coordinado por Ayala Marín (2013) revelaba que "se sigue representando a las mujeres en sus relaciones familiares, es 
decir, como esposas y madres, sobre todo" (p. 77). Sin embargo, hay escenarios donde la participación de la mujer es cada vez más creciente, como en el periodismo. En el ámbito particular del periodismo deportivo, las féminas gradualmente han ganado espacio; aunque continúa restringido a unas pocas. Si bien en los inicios de las transmisiones deportivas no era común escuchar o ver a una mujer desempeñando el rol de periodista o comentarista, con el paso del tiempo se comenzó a incluir la imagen femenina.

Aunque es innegable que al principio, las mujeres cumplían en el periodismo deportivo un rol estético, actualmente muchas de las que lo practican se destacan por sus conocimientos y preparación en las carreras de Comunicación Social. Al emitir un comentario lo hacen con fundamento, demostrando así la preparación obtenida en las aulas universitarias.

Ante la pluralidad de gustos deportivos existentes en la sociedad, que deben ser atendidos por los medios con opciones de diversidad, es oportuna la presencia de las mujeres en espacios de información deportiva que anteriormente les estaban vetados. De esta manera, la incursión de las mujeres en el periodismo deportivo supera mitos establecidos en décadas pasadas, demostrando que son capaces de empoderarse en un ámbito comunicativo considerado exclusivo de los hombres.

\section{Metodología}

Para desarrollar el presente estudio se empleó el Método Científico. Izquierdo Arellano (2006, p. 101) lo define como un conjunto de actividades y procedimientos lógicamente sistematizados que el investigador utiliza para descubrir la verdad y enriquecer la ciencia. Este método se basa además en las teorías experimentales, las operaciones lógicas y la imaginación racional. 
La utilidad de este método consistió en que permitió determinar las razones, causas y consecuencias del por qué los medios de comunicación social de la localidad de Loja no dan la oportunidad a las mujeres para que realicen periodismo deportivo. De igual manera, contribuyó a verificar objetivos y contrastar hipótesis, luego de los resultados del trabajo de campo.

El Método Inductivo también se empleó. Este permite pasar de los hechos obtenidos mediante observaciones, comparaciones, abstracciones y experimentaciones al planteamiento de leyes, principios y teorías a través de un proceso de análisis que permite llegar a la síntesis (Sandoval, 2003, p. 6667). En el estudio, este método contribuyó a realizar un análisis profundo y exhaustivo, tomando en consideración hechos particulares del por qué la mujer lojana no realiza periodismo deportivo tanto en medios locales como nacionales, es decir, desde un aspecto específico para entrar a una cuestión más amplia.

Por su parte, el Método Deductivo sigue un proceso sintético y un medio analítico contrario al anterior; se presentan conceptos, definiciones, leyes y normas generales, de las cuales se extraen conclusiones y consecuencias particulares, sobre la base de afirmaciones generales presentadas (Barrios, 2003, p. 29).

Este método ayudó a estudiar teorías generales para determinar de situaciones particulares, sintetizando y analizando principios relacionados con las teorías vinculadas al periodismo deportivo y sobre todo estudios anteriores de la participación de la mujer en esta rama, así como en los medios de comunicación social; por lo tanto, permitió encontrar alternativas que beneficien a todas las personas en calidad de periodistas deportivos, debido a que todas las disciplinas deportivas mueven a la colectividad. 
Se asumió la perspectiva cualitativa de investigación, pues su propósito es explicar los fenómenos estableciendo regularidades en los mismos mediante la formulación de leyes generales que explican el comportamiento social. Así, el conocimiento debe fundarse en el análisis de los hechos reales, de los cuales debe realizar una descripción lo más neutra, lo más objetiva y lo más completa posible (Monje, 2011, p. 12).

Por tanto, esta perspectiva permitió estructurar resultados matemáticos o estadísticos resultantes del trabajo de campo, con los análisis e interpretaciones, conforme a lo arrojado en las técnicas de investigación aplicadas. Estas fueron la encuesta y la entrevista.

La encuesta ayudó a recoger información objetiva sobre quienes están involucrados o conocen de la problemática. Se estructuró un cuestionario como instrumento de medición que estuvo integrado por interrogantes cerradas y de opción múltiple. De un universo de 215 mil habitantes de las seis parroquias urbanas de Loja (El Valle, El Sagrario, Sucre, San Sebastián, Punzara y Carigán) -de acuerdo con los resultados del VI Censo de Población y VII de Vivienda realizado el 28 de noviembre de 2010-, se tomó una muestra representativa de 400 personas mediante la aplicación y el despeje de la fórmula para públicos infinitos, en virtud de que se trata de una población superior a los 100.000 habitantes (Sierra, 2005, p.352). Para ello se empleó la siguiente fórmula: 


$$
n=\frac{O^{2} \times p \times q}{E^{2}}
$$

Donde:

$\mathrm{N}=$ Universo a investigarse

$\mathrm{n}=$ Tamaño de la muestra

$\mathrm{O}^{2}=$ Nivel de confianza $(2)=4$

$E^{2=}$ Margen de error $(5)=25$

$p=$ Varianza (nivel de confiabilidad de que el fenómeno ocurra) $=50$

$q=$ Varianza (nivel no probabilística de que el fenómeno ocurra) $=50$

$4 \times 50 \times 50$

$\mathrm{n}=$

25

10000

$\mathrm{n}=$

25

$n=400$

En tanto, para la entrevista se elaboró un cuestionario con cinco interrogantes que fueron aplicadas a los directores de los programas deportivos UV Deportes y Deportivo 22 (los más sintonizados en Loja); así como a la Jefa de Redacción de Diario Crónica, al Gerente de Diario Centinela, al presentador de Deportivísimo y Entretiempo y al presidente del Círculo de Periodistas Deportivos de Loja. 


\section{Resultados y discusión}

El estudio arrojó resultados significativos en cuanto a la preferencia de los públicos lojanos para informarse sobre temas deportivos. Del total de encuestados, el 50\% (200 personas) se inclina por la televisión. Un 30\% (120) apuesta por la radio; mientras que un 20\% (80) prefiere la prensa. Estas cifras colegian que la mayoría de encuestados prefiere la televisión, pues este medio está acompañado de imágenes en movimiento y sonido. Puede inferirse que el gusto por la radio se determina porque mientras esta se escucha permite realizar otras actividades; en cambio, la prensa impresa requiere de concentración para ser leída, por lo que ocupa más tiempo.

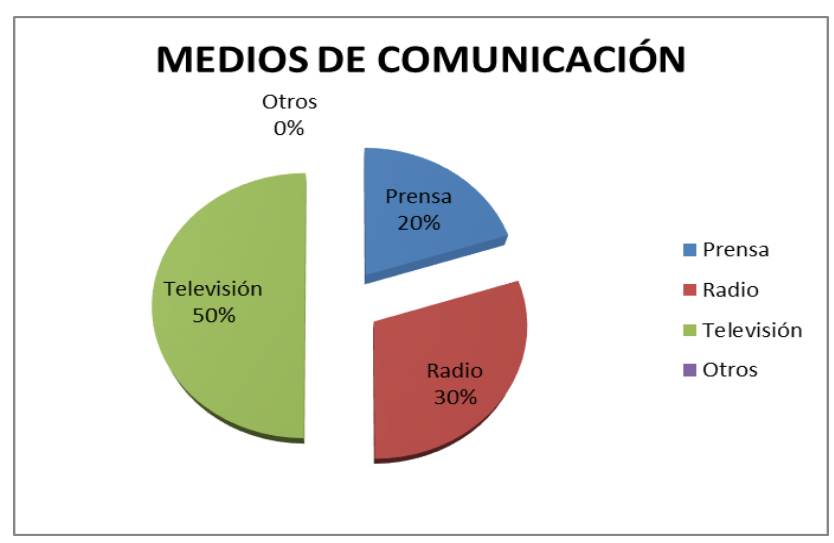

Figura 1: Medios que prefieren los lojanos para informarse sobre deporte.

Fuente: Elaboración Propia

Al indagar en torno a los programas deportivos audiovisuales que más agradan a los públicos, un 20\% (80 personas) declaró que en su preferencia está el programa televisivo Deportivo 22 y otro $20 \%$ (80) señaló a UV Deportes (televisión). Un 15\% (60) refirió que les agrada Deportivísimo (radio) y otro 15\% destacó a Contacto Deportivo que también se transmite por las ondas radiales. 
El 12.5\% (50) se apunta en Entretiempo (radio), mientras 8.75\% (35) aluden a Cronómetro y un porcentaje similar menciona a Adrenalina Deportiva, ambos radiales. La indagación realizada muestra que los programas deportivos que se transmiten en los medios locales son los de la radio, aunque es oportuno señalar que los que se emiten por la televisión regional también tienen alto reconocimiento entre los encuestados. La radio lojana, sin embargo, diversifica las ofertas sobre deporte, de ahí la mención a distintos programas deportivos en este medio de comunicación.

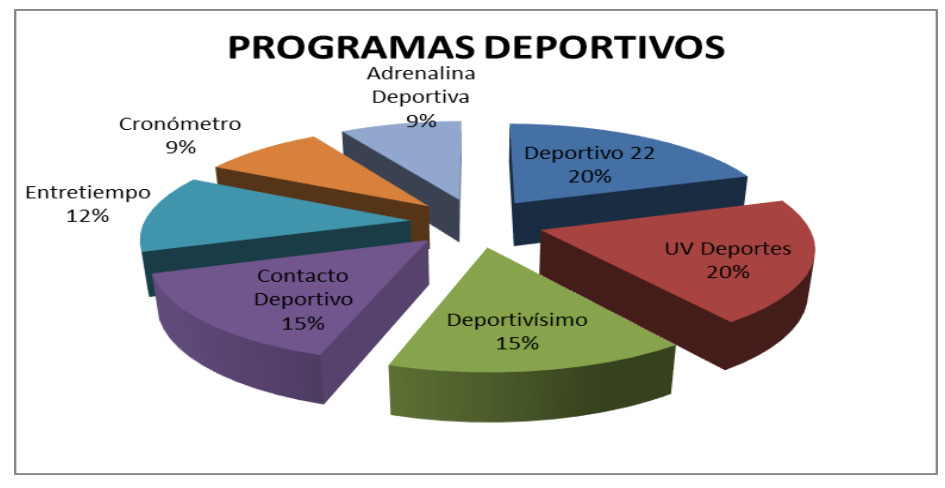

Figura 2: Programas deportivos que prefiere el público lojano. Fuente: Elaboración Propia

Igualmente, la totalidad de encuestados refirió que el periodismo deportivo lojano se realiza sólo por hombres, que son quienes gozan de mayores oportunidades. Esto se debe al predominio de estereotipos, pues todos los programas son patrocinados 0 conducidos por varones, existiendo discriminación hacia las mujeres que gustan de esta especialización del periodismo; por lo tanto las oportunidades son mínimas o simplemente no existen para el sexo femenino. La interrogante fue propicia para indagar 
posteriormente en consideraciones sobre la presencia de la mujer en los programas deportivos que se transmiten en los medios de comunicación locales $\mathrm{y}$, mientras un 75\% (300 personas) respondió no tener inconvenientes en que un programa sobre deportes incluya mujeres periodistas para dirigir 0 conducir las secciones, el 25\% (100) restante mostró inconformidad por asegurar que las mujeres no están aptas para asumir el rol de entrevistar o informar en un programa especializado en deportes.

Sin embargo, el presidente del Círculo de Periodistas Deportivos de Loja Washington González Estrella refirió en entrevista para el estudio que "las mujeres tienen todo el potencial académico e intelectual para dirigir un programa deportivo, su voz se vuelve exclusiva en un medio donde por tradición solo se ha escuchado a varones" (W. González, comunicación personal, 8 de febrero de 2017). Y es que, como refiere José Íñiguez, director de UV Deportes, "hoy, más allá de intentar remozar los programas deportivos con figuras femeninas, se cuenta con el aporte y talento de esas que han logrado incursionar con mucha preparación. Sin dudas, es una gran contribución para el periodismo deportivo" (J. Íñiguez, comunicación personal, 13 de marzo de 2017).

Como puede verse en la figura 3 , existen varias causas que los públicos lojanos reconocen como limitantes para la participación de la mujer en el ejercicio del periodismo deportivo. 


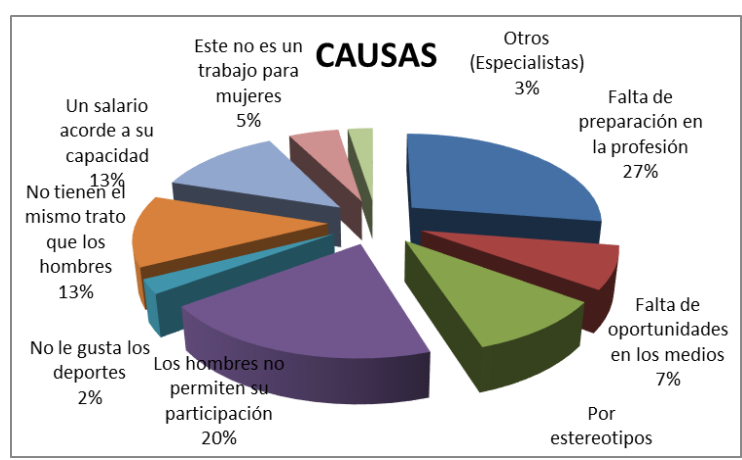

Figura 3: Causas de la nula participación de la mujer en el periodismo deportivo. Fuente: Elaboración Propia

Varios son los motivos que los públicos lojanos avalan como límites para que las mujeres se especialicen en periodismo deportivo. Las más significativas son precisamente la falta de preparación en la profesión (reconocida por 110 personas, que representan el 27\%) y el hecho de que los hombres no permiten que las mujeres participen (señalada por 80 personas, lo que equivale al $20 \%$ ). En el primer caso, es oportuno reconocer que aunque en muchas universidades de Ecuador se ofrece la materia de Periodismo Deportivo, no ocurre así en la Universidad Nacional de Loja donde imparte la carrera de Comunicación Social. Esto puede constatarse en la malla curricular de la especialidad, visible en el sitio web de la universidad. De ahí que las egresadas de este centro de estudios superiores que se especialicen en deporte en instituciones mediáticas fuera de Loja, lo hacen por la pasión que sienten por el tema.

Esta idea la refuerza Luis Cabrera, productor del programa Deportivo 22, cuando asegura que "las escuelas de comunicación deben ofrecer espacios para dar a conocer sobre periodismo deportivo, de esa manera, esta área irá ganado adeptos para que un futuro (las mujeres) ejerzan la profesión con 
conocimientos en este campo" (L. Cabrera, comunicación personal, 5 de marzo de 2017).

En cuanto al segundo caso, José Íñiguez apunta tres aspectos que condicionan, a su juicio, por qué los hombres no permiten que las mujeres incursionen en el periodismo deportivo. En primer lugar, señala que aún persisten remanentes culturales en los imaginarios de los periodistas, directores y productores masculinos en torno a que el área deportiva no es un buen espacio de especialización para las féminas, pues por lo general ha sido ocupado en su mayoría por hombres. Esta idea también la sustenta Joffrey Íñiguez, director de Entretiempo, cuando asegura que "el problema radica en que prima el egoísmo de algunos administradores de los medios de comunicación o de quienes manejan los programas deportivos, que no dan cabida para la participación de la mujer" (J. Íñiguez, comunicación personal, 13 de marzo de 2017).

En segundo lugar, el director de UV Deportes considera que la remuneración económica que reciben los hombres por hacer periodismo deportivo es superior a la que reciben las mujeres, pues

... aún hay una concepción machista en el ámbito del deporte, descartando la posibilidad de que las damas puedan hacerlo. Tradicionalmente, en esta sociedad machista el hombre ha tenido supremacía, supuestamente por su mayor capacidad e inteligencia. Por lo tanto recibe mejor remuneración, lo cual no ocurre así con las damas. Entonces la remuneración actual no es justa ni equilibrada y eso posiblemente aleje a las señoritas para que incursionen. (J. Íñiguez, comunicación personal, 13 de marzo de 2017)

Otro aspecto que señala José Íñiguez se relaciona más con las preferencias de las mujeres por esos temas, pues muchas no ven al periodismo deportivo como 
un ámbito para desarrollarse intelectualmente. Por eso quizás prefieran incursionar en otros campos informativos.

En este sentido, entre los encuestados existen diversos criterios en cuanto al éxito de un programa deportivo en relación con el género de sus conductores. Un 25\% (100) apuesta por la exclusividad de los hombres al conducir programas de corte deportivo. Mientras, un 37.5\% (150) asegura que este tipo de programa puede ser conducido exclusivamente por mujeres, en tanto similar porcentaje defiende la presencia de representantes de ambos sexos.

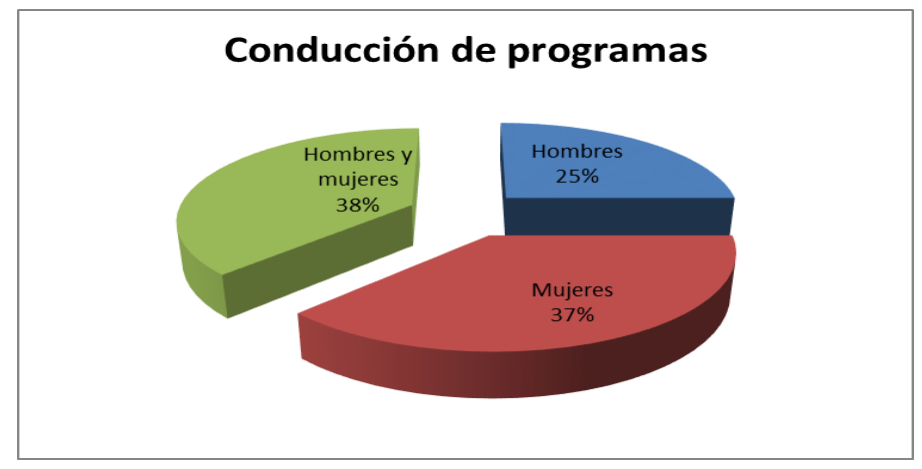

Figura 4: Preferencias de género para la conducción de programas deportivos.

Fuente: Elaboración propia

Como se observa, existe un equilibrio entre quienes opinan que las mujeres pueden conducir solas un espacio deportivo y los que consideran que deben hacerlo acompañadas por hombres. Con estas aseveraciones, se continúa reconociendo que los hombres deben estar al tanto de lo que comentan las mujeres en estos espacios, a la vez que desde el punto de vista dramático es importante el contraste de voces. Como recuerda Washington González Estrella:

Anteriormente a la voz femenina se la involucraba en las noticias y la farándula, pero hoy en el periodismo deportivo juega un rol demasiado valioso la voz 
femenina. No solo debe emplearse para presentar la noticia o estar en el diario vivir del acontecimiento informativo, sino para visitar lugares de entrenamiento, entrevistar autoridades y vivir diariamente con intensidad la información deportiva ya sea en fútbol o baloncesto, involucrándose directamente en el acontecer deportivo. (W. González, comunicación personal, 8 de febrero de 2017)

No obstante, el $75 \%$ de los encuestados (300) afirma que considera importante que en el periodismo deportivo exista equidad de género, abogándose por evitar la discriminación desde los puntos de vista social, económico, religioso, cultural o político, a tono con lo que señala la Constitución ecuatoriana (2008) en el artículo 36 referido a las Garantías Laborales de la Mujer: "El Estado propiciará la incorporación de las mujeres al trabajo remunerado, en igualdad de derechos y oportunidades, garantizándole idéntica remuneración por trabajo de igual valor. Todos tienen los mismos derechos e igualdades de oportunidades".

Los entrevistados para este estudio también reconocen los logros de las mujeres en todos los ámbitos de la sociedad que avalan su capacidad para desempeñar el periodismo deportivo. Luis Cabrera reconoce el estatus de igualdad en que se encuentran hombres y mujeres para ejercer el periodismo deportivo, pues "en los últimos tiempos las mujeres han logrado muchas hazañas y poco a poco van alcanzando puestos importantes en la sociedad" (L. Cabrera, comunicación personal, 5 de marzo de 2017).

En esta misma línea de pensamiento, Joffrey Î́niguez afirma que

El periodismo deportivo, puede ser manejado por todos o por quienes les gusta, pues la capacidad y responsabilidad es una característica de los profesionales de la comunicación social, por lo tanto no se debería hablar ni 
tener a menos a las mujeres, aunque desgraciadamente la sociedad esté cargada de estereotipos, sobre todo en el mercado local. La sapiencia es de todos, por ello no deberían existir individualidades, porque nadie es mejor que el otro son capacidades que deberían ser manejadas para el servicio colectivo y no personalmente. (J. Íñiguez, comunicación personal, 4 de abril de 2017) Gonzalo Piña (Comunicación personal, 2 de febrero de 2017) profundiza más en esta reflexión al alertar que los hombres se especializan en informar sobre deportes trascendentales como el fútbol, el baloncesto o el boxeo -por poner algunos ejemplos-, mientras las mujeres buscan especializarse en áreas deportivas que no son lo suficientemente abordadas por los hombres como la natación o la gimnasia, contribuyendo así a romper con el silenciamiento informativo que puede producirse en torno a estos deportes.

Finalmente, en la encuesta aplicada, el 75\% (300) de los encuestados reconoció la necesidad de diseñar una propuesta alternativa que permita insertar a la mujer en el periodismo deportivo lojano, con la finalidad de que se puedan poner en práctica y se constituyan en un aporte significativo para la comunidad y cambiar los pensamientos estereotipados que persisten en medios locales en relación con el trabajo que desempeñan las mujeres en el ámbito periodístico, y especialmente deportivo.

Según los entrevistados, una estrategia para insertar a mujeres periodistas en el ámbito deportivo debe estar matizada por la motivación. Washinton González insiste en la necesidad de "invitarlas, llamarlas, hacer casting, concursos... porque pueden existir damas a las que les gusta la narración, y eso no lo he visto yo al menos en nuestro país. Sería algo histórico" (W. González, comunicación personal, 8 de febrero de 2017). Asimismo, Gonzalo Piña (Comunicación personal, 2 de febrero de 2017) alerta que a las mujeres se les 
deben abrir las puertas de los programas deportivos no sólo a aquellas que ya han recibido un título universitario sino a las jóvenes que se forman como periodistas en la universidad lojana. En esto coincide también la Jefa de Redacción del Diario Crónica Ruth Abarca (Comunicación personal, 5 de febrero de 2017), para quien los medios deben confiar en que las mujeres tienen capacidad para laborar en cualquier ámbito de especialización dentro del periodismo.

\section{Conclusiones}

Aunque en los programas deportivos que se transmiten por los medios de comunicación de la ciudad ecuatoriana de Loja, solamente participa el género masculino, existe una tendencia generalizada a reconocer el valor de la mujer como profesional capaz de especializarse en el periodismo deportivo. De esta manera, poco a poco se produce una ruptura con los estereotipos que ha impuesto la sociedad en cuanto al ejercicio del periodismo deportivo como un área exclusiva de hombres. El hecho de que las mujeres periodistas dirijan o conduzcan espacios deportivos, daría un toque distinto a la programación existente en la ciudad. Por otro lado, la escasa preparación de las mujeres dentro del periodismo deportivo recibida en la academia, a pesar de que sería una limitante para su cabal desempeño, podría superarse si se les ofertaran oportunidades en los distintos espacios informativos dedicados a este particular, pues un programa deportivo tendría mayor audiencia si estuviese también conducido por mujeres, debido a que la voz y la imagen femeninas cautivan y atraen. 


\section{Bibliografía}

Alcoba López, A. (2010). ¿Quo vadis deporte? Del fracaso olímpico al triunfo de la política y capitalismo deportivo. Madrid: Esteban Sanz Editorial.

Ayala Marín, A. (2013). Justicia de género en medios de comunicación ecuatorianos.

Estereotipos y representaciones sobre las mujeres. Quito: CIESPAL.

Barrios, A. (2003). Metodología de la Investigación II. Guayaquil: Universidad de Guayaquil.

Billings, A. (2010). La comunicación en el deporte. Barcelona: Editorial UOC Constitución de la República de Ecuador (2008). Recuperado de www.lexis.com.ec González, Y. (2013). Terminología y polisemia en torno a la Educación Física. Viref,

Revista de Educación Física, Universidad de Antioquia, 2(4), 25-32.

Hernández, N. (2003). El lenguaje de las crónicas deportivas. Madrid: Cátedra. Izquierdo Arellano, I. (2006). Investigación Científica. Loja: Cosmos.

Kirby Ruiz, M. A. (2009). Incorporación de un segmento deportivo en las cuatro emisiones del informativo del canal 4; U.V Televisión de la Ciudad de Loja (Tesis de licenciatura), Universidad Nacional de Loja, Loja, Ecuador.

Lara, M. (2007). Periodismo deportivo: la fantasía de un oficio hecho profesión.

Revista Comunicología, 6. Recuperado de

http://revistacomunicologia.org/index.php?ltemid=73\&id=158\&option=co m_content\&task=view

Malla Guamán, J. M. (2017). La inserción de la mujer dentro del periodismo deportivo lojano, un reto comunicacional para lograr la igualdad de género y su influencia en el contexto sociocultural. Periodo mayo-octubre de 2014. propuesta alternativa (Tesis de licenciatura), Universidad Nacional de Loja, Loja, Ecuador. 
Molina Vizcarra, J. (2009). Periodismo deportivo 3.0. Razón y Palabra. Primera Revista Electrónica en América Latina especializada en Comunicación, 69. Recuperado de http://www.razonypalabra.org.mx/N/n69/Javier\%20Molina\%20Vizcarra.html Monje Álvarez, C. A. (2011). Metodología de la Investigación Cuantitativa y Cualitativa. Guía Didáctica. Neiva: Universidad Surcolombiana.

Moragas, M. (2008). Comunicación y deporte en la era digital. Actas Comunicación y deporte. IX Congreso A.E.I.S.A.D. Investigación Social y Deporte, 8. Madrid: Librerías Deportivas Esteban Sanz.

Moreno Guano, J. A. (2010). La inserción de la mujer en el periodismo deportivo. Acciones para incentivarlo en la sociedad ecuatoriana actual (Tesis de licenciatura), Universidad Técnica de Cotopaxi, Ecuador.

Paniagua Santamaría, P. (2003). Información deportiva: especialización, género y entorno digital. Madrid: Fragua.

Sandoval, E. (2003). Metodología de la Investigación Científica. Cuenca: Don Bosco.

Sierra Bravo, R. (2005). Métodos de investigación en Ciencias Sociales. Madrid: Paraninfo.

Sobrados León, M. (2005). Entre la información y la opinión. Una revisión de los principales géneros en el periodismo deportivo, en Marín Montín, J. (Ed.). Comunicación y Deporte. Nuevas perspectivas de análisis. Sevilla:

Comunicación Social Ediciones y Publicaciones. 